\title{
Development of Synchrotron DSC/WAXD/SAXS Simultaneous Measurement System for Polymeric Materials at the BL40B2 in SPring-8 and its Application to the Study of Crystal Phase Transitions of Fluorine Polymers
}

\author{
Hiroyasu Masunaga, ${ }^{1, \dagger}$ Sono Sasaki, ${ }^{1}$ Kohji Tashiro, ${ }^{2}$ Makoto HanesaKa, ${ }^{2}$ \\ Masaki TAKATA, ${ }^{1,3}$ Katsuaki InOUE, ${ }^{1}$ Noboru OHTA, ${ }^{1}$ and Naoto YAGI ${ }^{1}$ \\ ${ }^{1}$ Japan Synchrotron Radiation Research Institute (JASRI)/SPring-8, \\ 1-1-1 Kouto, Sayo, Sayo 679-5198, Japan \\ ${ }^{2}$ Department of Future Industry-oriented Basic Science and Materials, Graduate School of Engineering, \\ Toyota technological Institute, Tempaku, Nagoya 468-8511, Japan \\ ${ }^{3}$ The RIKEN Harima Institute/SPring-8, 1-1-1 Kouto, Sayo, Sayo 679-5198, Japan
}

(Received July 20, 2007; Accepted September 1, 2007; Published October 31, 2007)

\begin{abstract}
A new system of rapidly-scanning DSC/WAXD/SAXS simultaneous measurements has been successfully developed at the BL40B2 in SPring-8, Japan. The combination of the ultra-bright and highly-directional X-ray beams with the highly-sensitive two-dimensional (2D) detectors such as an imaging plate (IP), a CCD detector and a flat panel (FP) detector has made it possible to perform the rapidly-scanning time-resolved measurements of WAXD and SAXS for the polymeric materials subjected to the various kinds of external field like temperature, tensile force, magnetic field, hydrostatic pressure, and so on. The experimental data about the phase transitions of vinylidene fluoridetrifluoroethylene copolymer with VDF $82 \mathrm{~mol} \%$ content and poly(vinylidene fluoride) form I have been presented as actual examples, where the phase transitions of the polar crystal consisting of planar-zigzag chains to the gauche-type high-temperature phase have been confirmed definitely and the remarkable change in lamellar stacking structure could be detected in the temperature region immediately below the melting point. [doi:10.1295/polymj.PJ2007105]

KEY WORDS Simultaneous Measurement System / Wide-angle X-ray Diffraction / Small-angle X-ray Scattering / Differential Scanning Calorimetry / SPring-8 / Poly(vinylidene fluoride) / Phase Transition /
\end{abstract}

Polymeric materials consist of complicated aggregation state of chains. In the case of semicrystalline polymer, for example, these chains gather together to form the crystalline region or a crystalline lamella. These lamellae are stacked together to construct a spherulite. There are amorphous region between these stacked lamellae. In the spherulite, the lamellae extend and twist periodically from the spherulitic center along the radial direction, giving the so-called extinction ring in its polarized optical microscopic image. It is essentially important to clarify the evolution process of this complicated hierarchical structure of polymers from the various viewpoints on angstrom-tomicrometer scale in order to design and control their physical and structural properties. One of the most useful and quantitative methods for attainment of this purpose is to use the $\mathrm{X}$-ray scattering technique. The wide-angle X-ray diffraction (WAXD) gives us information on the chain conformation and the aggregation state of chains in the crystalline and amorphous re- gions. The small-angle X-ray scattering (SAXS) tells us about the rough form of molecular chains, the aggregation structure of crystalline lamellae, and so on. Therefore, the time-resolved measurements of WAXD and SAXS allow us to trace the structural evolution process of polymers in the heating or cooling process, for example. There were many reports on the WAXD and/or SAXS experimental data. ${ }^{1}$ In most cases the WAXD or SAXS measurement was performed for a sample separately by assuming the reproducibility of the experimental conditions. But, strictly speaking, we do not have any guarantee about the reproducibility of the experimental conditions in the WAXD and SAXS measurements, since the polymeric material has a large hysteresis concerning the thermal or mechanical treatment. It is more ideal to perform the simultaneous measurement of WAXD and SAXS for one sample subjected to the completely same conditions. The WAXD and SAXS simultaneous measurements, and those combined with the other

${ }^{\dagger}$ To whom correspondence should be addressed (Tel: 0791-58-0750, Fax: 0791-58-0830, E-mail: masunaga@spring8.or.jp). 
type of measurements have been carried out by many researchers. ${ }^{1,2}$ However, X-ray scatterings from polymeric materials are relatively weak in intensity because the main chemical component of polymers generally consists of the light elements, so that it is quite difficult to perform the rapid measurement of SAXS from polymeric materials in laboratory-level experiments. In other words, it is hard to trace the structural evolution process through the time-resolved simultaneous measurement of WAXD and SAXS as long as the X-ray instrument on the laboratory level is used. $\mathrm{X}$-Ray beams originated from synchrotron radiation source may solve this dilemma quite nicely. The combination of the ultra-bright X-ray beams with highlysensitive detectors makes it possible to perform the above-mentioned in situ WAXD/SAXS simultaneous measurements satisfactorily. ${ }^{3-21}$ In the European Synchrotron Radiation Facility (ESRF), ${ }^{6}$ The Advance Photon Source (APS), ${ }^{7,8}$ and the other synchrotron radiation facilities, for example, the WAXD/SAXS simultaneous measurement system with two-dimensional (2D) detectors (2D-WAXD/SAXS measurement system) is a default utilized for many general users, helping them to create many papers on the structural evolution process of polymers. In SPring-8 and Photon Factory, the WAXD/SAXS simultaneous measurements were carried our by the limited numbers of technically-skilled particular researchers. ${ }^{13-17,20,21}$ As mentioned above, the 2D-WAXD/ SAXS measurement system is a necessary tool for kinetic investigations on the hierarchical structure of polymers.

Synchrotron radiation produced in SPring-8 is characterized by the highest brilliance in the world and high directionality. Therefore, utilization of synchrotron-sourced $\mathrm{X}$-rays as the incident beams is quite effective to perform time-resolved X-ray scattering measurements for polymeric materials and to detect $\mathrm{X}$-ray scatterings from the narrow and/or thin areas of polymeric materials with the high $\mathrm{S} / \mathrm{N}$ ratio and special resolution. Yagi and Inoue performed the WAXD/SAXS measurements to test the ability of a new type detector called a CMOS FPD at the BL40XU and the BL40B2 in SPring- $8 .{ }^{21}$ Also, Amemiya et al. reported the experimental results obtained using a FP detector. ${ }^{15}$ Ito et al. reported the WAXD/SAXS data of biodegradable polymers measured with the taper optics fiber CCD detector as a detector for WAXD. ${ }^{20}$ However, these systems were optional at each beamline. Therefore, it was an indispensably important theme for us to supply the rapidlyscanning 2D-WAXD/SAXS measurement system for the common use. The addition of various techniques to this simultaneous system can make it possible to perform more highly sophisticated type of experi- ment; for example, the 2D-WAXD/SAXS combined with each of the Raman spectrum measurement, the differential scanning calorimetetry (DSC), and so on.

In the present paper we will report about the development of a 2D-WAXD/SAXS measurement system for polymeric materials at the BL40B2 in SPring-8. This system has been combined with DSC equipment for the study of thermally-induced structural change in the heating or cooling process of polymers. As an application of the thus-built-up system, we have measured the temperature dependences of WAXD, SAXS and enthalpy change to investigate the crystal phase transition of fluorine polymers for the purpose to clarify the relationship between the crystal structure change and the morphological change occurring in the ferroelectric phase transition of vinylidende fluoride-trifluoroethylene (VDF-TrFE) copolymers including the crystal form I of poly(vinylidene fluoride).

\section{SETTING UP OF DSC/WAXD/SAXS SIMULTANEOUS MEASUREMENT SYSTEMS}

Figure 1 shows a schematic explanation and photographs of the DSC/WAXD/SAXS measurement systems built at the BL40B2 in SPring-8. The BL40B2 was basically designed as a SAXS (solution scattering) beamline for the structural analysis of proteins. The light source of the BL40B2 is generated by a bending magnet of $0.679 \mathrm{~T}$ and shows critical energy of $28.9 \mathrm{keV}$. The generated white X-rays are monochromatized using a fixed-exit double Si crystal mon-

(a)

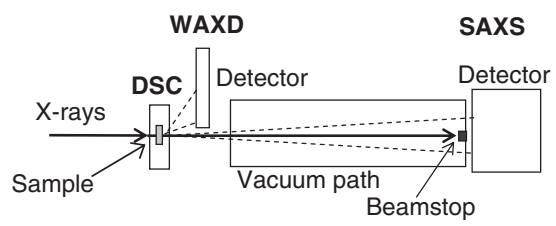

(b)

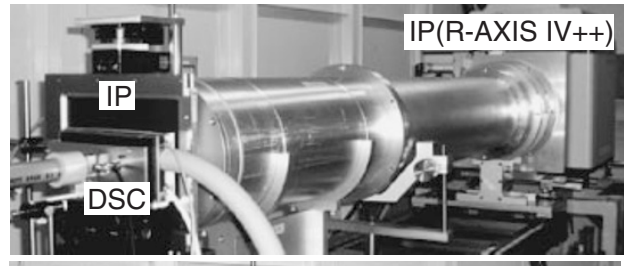

(c)

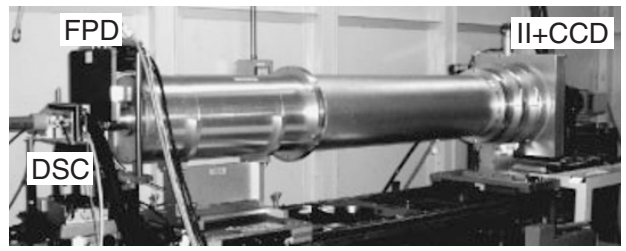

Figure 1. Schematic illustration and photograph of the DSC/ WAXD/SAXS simultaneous measurement system built-up at the BL40B2 in SPring-8. (a): DSC/WAXD/SAXS experimental geometry; (b) The system with imaging plates detectors; (c) The system with a FD detector and II + CCD detector. 
ochromator and focused by a $1 \mathrm{~m}$-long rhodium-coated bent cylinder mirror. ${ }^{22}$ In the experimental hutch of the BL40B2, the space around a sample stage is large enough to place comparatively large equipments, such as a drawing machine, a spinning machine, and an additional detector. We designed a holder of a detector for WAXD which has four adjustable axes. The holder was manipulated from the outside of the experimental hutch so that the detectable plane of the WAXD detector is perpendicular to the incident direction of the Xray beams. The dried chicken leg tendon collagen and cerium oxide were used as the standard references for SAXS and WAXD, respectively. The intensity of SAXS and WAXD was calculated for the large number of scattering patterns through our software at a time.

In Figure 1(a), two detectors for WAXD and SAXS were placed relatively close and far from a sample in a DSC vessel, respectively. A vacuum path was placed between the detectors. The distance from the sample to each detector (camera length) can manually be changed depending on the q-range $(\mathbf{q}: 4 \pi \sin \theta / \lambda$, $2 \theta$ : scattering angle, $\lambda$ : wavelength of incident $\mathrm{X}$ rays) of X-ray scattering the users will use for structural analyses.

Two kinds of setup have been made properly depending on the purpose of the experiments. Figure 1(b) indicates the DSC/WAXD/SAXS measurement system using $250 \mathrm{~mm} \times 200 \mathrm{~mm}$ and 300 $\mathrm{mm}$-square imaging plates (IP) has as detectors for both of WAXD and SAXS so that the 2D-scattering patterns in the high $\mathrm{S} / \mathrm{N}$ ratio can be obtained. The 2D-SAXS signal from the sample passed through a hole with an $8 \mathrm{~mm}$ diameter, which was opened at the center of IP (BAS-SR2025, Fujifilm Co., Japan) for WAXD. The IP for WAXD has to be changed manually, was read by BAS-2500 (Fujifilm Co., Japan). It is possible to perform the time-resolved $\mathrm{X}$-ray scattering measurements at an interval time between measurements of $c a .4 .5 \mathrm{~min}$ using the R-AXIS IV++(Rigaku Co., Japan). This system allows us to obtain the scattering intensity in the dynamic range of 5 and 6 orders for WAXD and SAXS, respectively. The pixel resolution of the IP for WAXD is $50 \mu \mathrm{m}$ per pixel and that for SAXS is and $100 \mu \mathrm{m}$. IP system a detector for SAXS. In Figure 1(b), the camera length was $c a .40 \mathrm{~mm}$ and $2 \mathrm{~m}$ for WAXD and SAXS, respectively with the wavelength of $0.12 \mathrm{~nm}$, so that $\mathrm{q}=$ $8-25$ and $\mathrm{q}=0.06-2 \mathrm{~nm}^{-1}$ were covered by WAXD and SAXS detectors, respectively.

Figure 1(c) shows another system using a CMOS FP detector (C9728DK, Hamamatsu Photonics K. K., Japan) which is an active-pixel detector with $1056 \times$ 1056 pixels $(52.8 \mathrm{~mm} \times 52.8 \mathrm{~mm})$ and a CCD (charge-coupled device) detector (C4880-50-24A,
Hamamatsu Photonics, K. K. $)^{23}$ with X-ray imaging intensifier (V4554P, Hamamatsu Photonics, K. K.) (II + CCD) as detectors for WAXD and SAXS, respectively. The pixel resolutions of the detectors for WAXD and SAXS are $50 \mu \mathrm{m}$ and c.a. $150 \mu \mathrm{m}$, respectively. The linearity of this FP detector was observed with an increase in exposure time, up to the pixel value of 14-bit reached. The dynamic range of detected scattering intensity is 3 and 4 orders for WAXD and SAXS, respectively. In this system, the more rapid change of the hierarchical structure of polymeric materials can be traced. The time-resolved measurement can be performed every few seconds although the reading-out time (c.a. $5 \mathrm{~s}$.) of the II + CCD has to be added in every measurement. The data collection timing can be controlled by an external trigger which can synchronize the FD detector and II + CCD detector with the external instrument such as a stretcher, a temperature controller, and etc. The measured q-range in Figure 1(c) was almost same as that in Figure 1(b).

In the present work, the thermal data and X-ray scatterings were measured simultaneously by combining the WAXD/SAXS detector with the DSC equipment. ${ }^{23-25}$ An FP84HT hot stage and an FP90 control unit (Mettler-Toledo International Inc., U.S.A.) were utilized for DSC measurements. ${ }^{24-26}$ The schematic illustration of the DSC vessel and the sample cell is shown in Figure 2. The detector-side window of a vessel of the hot stage was customized in opening an angle for measuring the X-ray scattering up to the scattering angle $28^{\circ}$. For an unoriented sample, a bottom plate of a standard aluminum sample pan was changed to thinner one to detect SAXS without anisotropic scattering from the pan. In the case of an oriented sample, the sample was set into a glass capillary to keep the oriented direction of the sample. The capillary was sandwiched between the base and cover plates of the vessel of the FP84HT hot stage to fix its position. Thus prepared sample was sandwiched between the base and cover plates of the vessel to keep

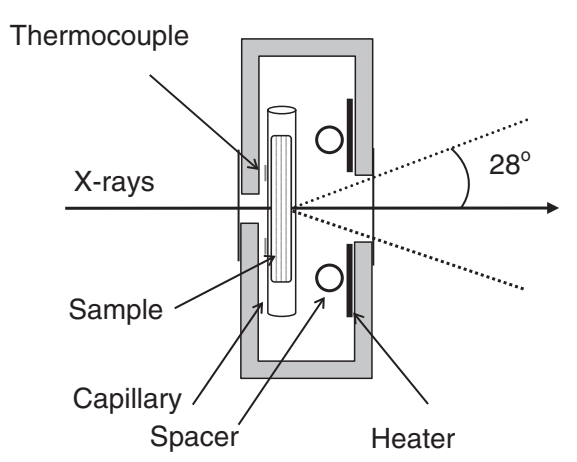

Figure 2. A schematic illustration of DSC cell used for the oriented sample set in a capillary. 
the pan from dropping down to the ground during measurements. Ring-type insulation placed between the sample pan and a cover plate of the vessel pushed the sample pan to contact perfectly to the thermocouple set on the base plate of the vessel. This insulation showed no signal in DSC thermograms in a temperature range from 30 to $300^{\circ} \mathrm{C}$.

We will present below the results of WAXD/ SAXS/DSC measurement experiments concerning the phase transition of vinylidene fluoride copolymers and poly(vinylidene fluoride) (PVDF) itself as the examples of application of the thus developed simultaneous system in SPring-8.

\section{PHASE TRANSITION OF UNIAXIALLY- ORIENTED VDF 82\%-TRFE COPOLYMER}

Vinylidene fluoride-trifluoroethylene (VDF-TrFE) random copolymers are well known to show the ferroelectric phase transition between the polar and nonpolar crystalline phases in the heating and cooling processes. At low temperature the essentially planarzigzag chains are packed together in the crystal lattice with the $\mathrm{CF}_{2}$ dipoles arrayed in parallel to the $b$ axis. ${ }^{27-31}$ This polar crystal phase transfers to the nonpolar phase by heating above the Curie transition point, where the trans-zigzag chains change their conformation to the gauche-type form and these conformationally-disordered chains rotate violently around the chain axis in the pseudo-hexagonal cell to give the nonpolar crystal, so called high-temperature (HT) phase. ${ }^{27,28}$ The crystal structure of VDF-TrFE copolymer at low temperature is essentially the same as that of PVDF form I. PVDF form I must be prepared by stretching the unoriented form II sample, obtained by slowly cooling the melt, at room temperature. In contrast, it is relatively easier in the case of VDF-TrFE copolymers with VDF content lower than $75 \mathrm{~mol} \%$ to prepare the unoriented polar crystal form I even when the sample is cooled slowly from the melt. ${ }^{27-36}$ In the present study we focus our attention onto the VDFTrFE copolymer with VDF content higher than $80 \mathrm{~mol} \%$. In this case, however, the situation becomes similar to that of PVDF homopolymer. For example, VDF-TrFE copolymer with VDF 82\% (VDF82\%TrFE) sample crystallizes into form II of gauche-type chain conformation when cooled from the melt, just likely the case of PVDF. ${ }^{37}$ But, this form II is relatively unstable and transfers easily to the polar crystal form I when it is stretched at room temperature. Since form I crystal is a ferroelectric phase, it is useful to utilize this form I sample for the purpose to clarify an effect of TrFE comonomer on the ferroelectric transition behavior of VDF $82 \%$ copolymer in comparison with the transition behavior of form II sample. ${ }^{37}$

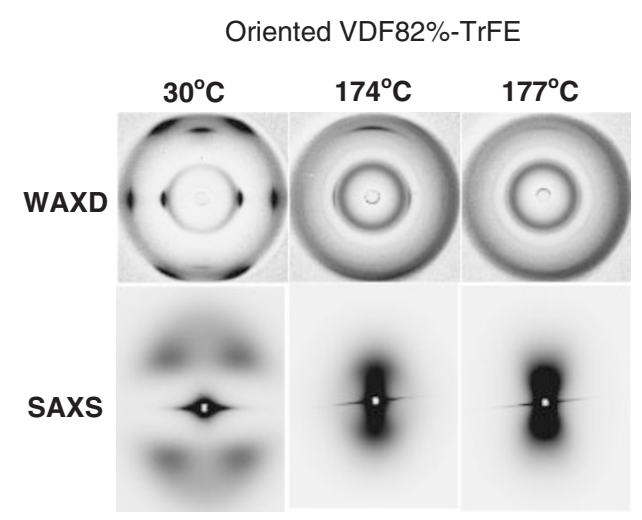

Figure 3. WAXD and SAXS patterns of an uniaxially-oriented VDF82\%-TrFE copolymer sample measured simultaneously at $30^{\circ} \mathrm{C}, 174^{\circ} \mathrm{C}$, and $177^{\circ} \mathrm{C}$ in a heating process. The meridional direction is parallel to the stretching direction of the sample.

The VDF82\%-TrFE copolymer sample was supplied by Daikin Kogyo Co., Japan. The sample was melted at $200{ }^{\circ} \mathrm{C}$ and rapidly quenched into water followed by stretching about 4 times the original length at room temperature to obtain the oriented form I sample. The sample set in a quartz capillary of 0.7 $\mathrm{mm}$ diameter was sandwiched between the cover plates of the DSC hot stage. The DSC stage was set in the beam line to measure the WAXD and SAXS simultaneously. The sample-to-imaging plate distances were 76 and $2170 \mathrm{~mm}$, respectively, for WAXD and SAXS. The X-ray wavelength was 0.12 $\mathrm{nm}$. The temperature was increased in a stepwise way, and the WAXD and SAXS were measured with the exposure time of $30 \mathrm{~s}$ at each temperature.

Figure 3 shows the 2-D WAXD and SAXS patterns measured for the uniaxially-oriented VDF82\%-TrFE sample in the heating process from room temperature. The WAXD pattern at $30^{\circ} \mathrm{C}$ corresponds to that of the oriented form I crystal. The SAXS pattern shows the four-points scattering pattern, indicating a tilt of stacked lamellae from the draw direction. As the temperature was increased, the form I reflections decreased in intensity and the degree of orientation became lower. The small angle scattering peaks shifted toward the lower angle side, and the tilting angle became smaller. At around $174{ }^{\circ} \mathrm{C}$ the SAXS pattern changed to the meridional two-points scattering pattern and the new reflections started to appear in the WAXD pattern. Figure 4 shows the X-ray diffraction patterns taken at $170^{\circ} \mathrm{C}$ and $177^{\circ} \mathrm{C}$, where the amorphous halo is subtracted from the original pattern to extract the reflections of HT phase more clearly. The X-ray diffraction pattern of this HT phase is similar to that of PVDF form II and/or form III.

The X-ray scattering profiles were obtained from these 2-D patterns and the peak intensity was plotted against the temperature as shown in Figure 5, where 


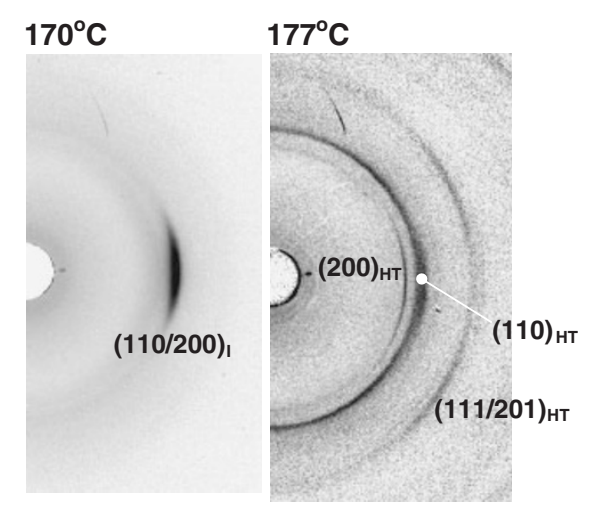

Figure 4. Enlarged X-ray diffraction patterns of VDF82\%TrFE measured at $170^{\circ} \mathrm{C}$ and $177^{\circ} \mathrm{C}$, where the contribution of amorphous hallo was subtracted.

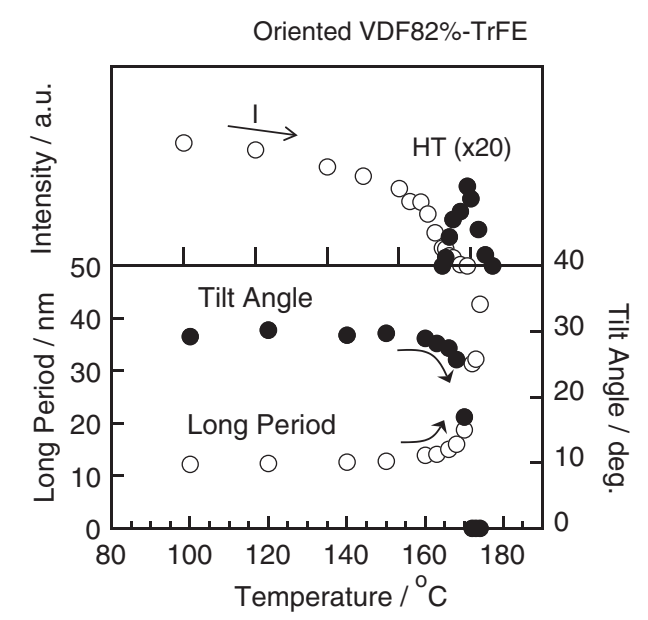

Figure 5. Temperature dependences of the peak intensity of the $(200 / 110)_{\mathrm{I}}$ and $(200 / 110)_{\mathrm{HT}}$ reflections and the long period and tilt angle estimated for an uniaxially-oriented VDF82\%-TrFE copolymer sample.

the reflections (110/200) of form I and (200) of HT phase were used. As the temperature increased the for$\mathrm{m}$ I reflections decreased in intensity and those of HT phase increased instead, showing a clear phase transition from polar form I to nonpolar HT phase in the temperature region near the melting point. At the same time the long period of stacked lamellar structure increased drastically and the lamellar tilting disappeared almost to zero. All of these observed phenomena are essentially the same as those reported for VDF $65 \%$ and $73 \%$ copolymers, ${ }^{38}$ indicating that the lamellar stacking morphology is affected sensitively by the structural change occurring in the crystalline lattice. The highly accelerated thermal motion of molecular chains, which is induced through the trans-gauche conformational exchange, enhances the growth of lamellar thickness along the chain axis, resulting in the standing up of tilted lamellae, as illustrated in Figure 6.

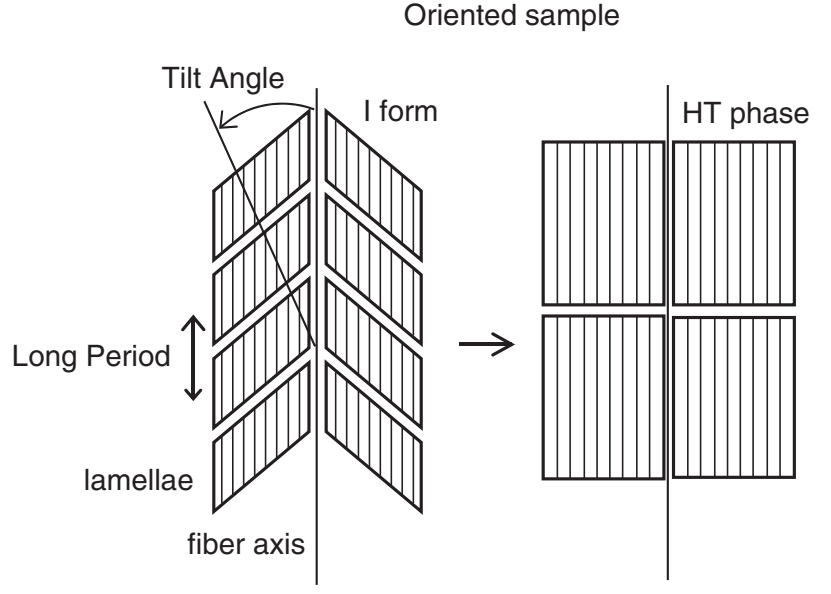

at low temperature just under a melting point

Figure 6. An illustration of the morphological change in the phase transition from form I to HT phase of oriented sample.

\section{MELT-SLOWLY COOLED VDF82\%-TRFE COPOLYMER}

In the preceding section, the phase transition of uniaxially-oriented form I sample of VDF82\% copolymer has been investigated to demonstrate the usefulness of simultaneous measurement system of DSC/ WAXD/SAXS. In this section, another example is shown, which is an application of simultaneous measurement system of triple instruments, DSC, WAXD and SAXS for the study of crystalline phase transition of unorientred VDF $82 \%$ sample. The simultaneous measurement of DSC and WAXD is now popular even in the usual laboratory level, but it is actually impossible to perform the simultaneous and rapid measurement of DSC and SAXS in addition to WAXD. The strong X-ray source of SPring-8 and highly-sensitive detectors (an imaging plate for WAXD and the RAXIS IV++ for SAXS) may satisfy this requirement and make it possible to perform the rapid DSC/ WAXD/SAXS simultaneous measurement in situ.

As mentioned above, the slow cooling of VDF $82 \%$ copolymer from the melt $\left(0.1{ }^{\circ} \mathrm{C} / \mathrm{min}\right)$ results in the formation of unoriented form II sample. The DSC/ WAXD/SAXS simultaneous measurement was performed at $5 \mathrm{~min}$ interval in the heating process at $1.0^{\circ} \mathrm{C} / \mathrm{min}$. The measurement was started by opening an X-ray shutter at constant time interval triggered by a signal from an R-AXIS IV++ controller. This trigger signal was used also for the control of the computer system of DSC. The exposure time was $30 \mathrm{~s}$ and the reading out time of imaging plate was $4.5 \mathrm{~min}$ for the R-AXIS IV++ system. Since the sample was unoriented, the observed WAXD and SAXS patterns consisted of rings. The 1-dimensional profiles were 

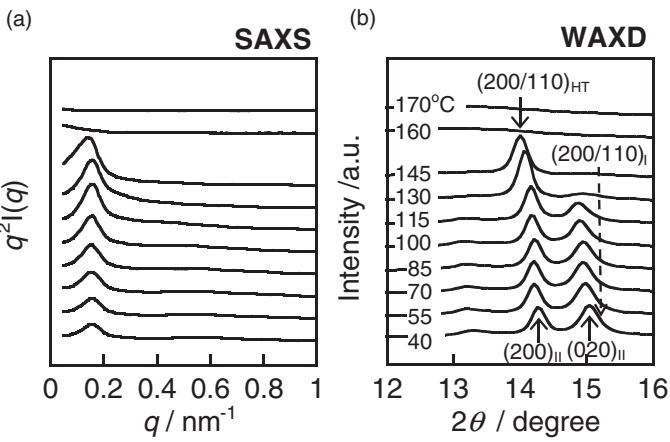

Figure 7. $\mathrm{SAXS}(\mathrm{a})$ and $\mathrm{WAXD}(\mathrm{b})$ profiles estimated for the melt-slowly-cooled VDF82\%-TrFE copolymer sample during heating process at a rate of $1.0^{\circ} \mathrm{C} / \mathrm{min}$.

calculated from these rings by integration along the azimuthal direction.

Figure 7 shows the WAXD and SAXS profiles obtained for the melt-slowly cooled VDF $82 \%$ sample in the heating process at $1.0^{\circ} \mathrm{C} / \mathrm{min}$. As the temperature was increased, the peak intensity of the $(020)_{\text {II }}$ at $2 \theta=15^{\circ}$ decreased remarkably at about $120^{\circ} \mathrm{C}$, where the subscript II indicated the reflection coming from the crystalline form II. The (200) II peak at $2 \theta=14^{\circ}$ shifted to lower angle side and changed apparently continuously to the reflection $(200 / 110)_{\mathrm{HT}}$ intrinsic of the HT phase. This X-ray reflection peak of the HT phase increased in intensity with temperature and disappeared above the melting point. Different from the phase transition from form I to HT phase observed for the various VDF-TrFE copolymer samples with different VDF contents, the X-ray reflection of the HT phase detected here was not sharpened even when the temperature was increased.

Figure 8 shows the temperature dependences of peak intensities of these observed reflections. The phase transition could be seen to occur from form II to HT phase in a wide temperature region of 100$160^{\circ} \mathrm{C}$. In this figure the intensity of X-ray reflection intrinsic to form I is also plotted, which started to decrease at around $120^{\circ} \mathrm{C}$ and the HT phase reflections increased in intensity furthermore in addition to the increment due to the transition from form II. The form II disappeared around $150^{\circ} \mathrm{C}$. The reflections of form I and HT phase disappeared in higher temperature region. During these transitions the long period estimated from SAXS data was found to increase from $40 \mathrm{~nm}$ to $45 \mathrm{~nm}$. (A long period was not able to be estimated although it seemed that the peak was sifting to a low angle.) As indicated in this figure, the several peaks detected in the DSC thermogram can be assigned to these phase transitions fairly reasonably. The shoulder observed in a region of 100 to $140^{\circ} \mathrm{C}$ might correspond to the transition from form II to HT phase. A large peak around $150^{\circ} \mathrm{C}$ might reflect

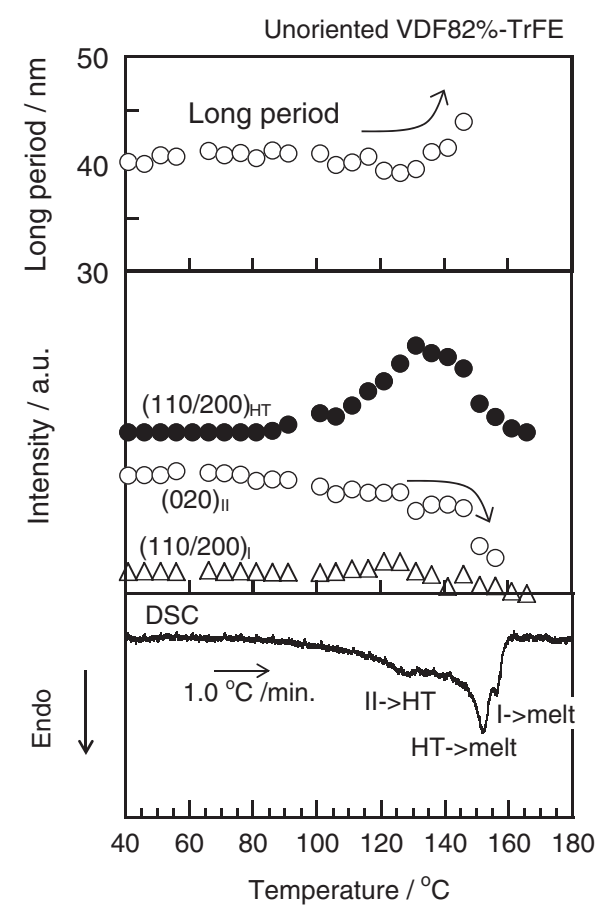

Figure 8. Temperature dependences of long period and peak intensity of the X-ray reflections for form I, form II and HT phase estimated for an uniaxially-oriented VDF82\%-TrFE copolymer sample in comparison with the corresponding DSC thermogram.

the transition of form I to HT phase and the meltings of form II and HT phase. A small shoulder at $155^{\circ} \mathrm{C}$ might correspond to the melting of form I and/or HT phase.

\section{CONFIRMATION OF CRYSTALLINE PHASE TRANSITION OF PVDF FORM I}

In the preceding sections the phase transition behaviors of VDF $82 \%$ copolymer samples were discussed. The uniaxially-oriented VDF $82 \%$ sample shows the phase transition from form I to the HT phase in the temperature region close to the melting point ${ }^{39,40}$ By comparing this experimental result with the previously published phase transition behaviors observed for a series of VDF-TrFE copolymers, it can be said that the phase transition from polar form I to nonpolar HT phase occurs at higher temperature as the VDF content becomes higher. These data allow us to speculate that PVDF form I may show a phase transition to the HT phase in much higher temperature region close to the melting point. Tashiro et al. investigated the phase transition of PVDF form I crystal by measuring the temperature dependences of WAXD and polarized infrared spectra and found the phase transition from form I to III in a temperature region immediately below the melting point. But, sometimes the samples were melted before the observation of the phase transition. We need to confirm the observation 
of form I -to- HT phase transition during the heating process at relatively fast rate to avoid the local sample melting. In addition, they did not measure any SAXS data for observing the change in morphology related with the phase transition phenomenon as detected for the VDF-TrFE copolymers. Unfortunately, it is difficult to perform the measurement of WAXD and SAXS in the heating process of form I because the uniaxially-oriented PVDF sample is easily cut down by heating near the melting point. We were challenged to perform the simultaneous DSC/WAXD/ SAXS measurement using the above-mentioned synchrotron system. In this experiment we used the PVDF sample of enough high molecular weight, which is hard to flow even above the melting point as likely the case of ultra-high molecular weight polyethylene, helping us to observe the phase transition without any breakage of the sample near the melting temperature.

The PVDF sample used actually for this experiment was KF7200 supplied by Kureha Co., Japan. The sample was melted at $200^{\circ} \mathrm{C}$ and rapidly quenched into ice-water bath, followed by stretching about 3 times the original length at room temperature. The sample was set in the DSC equipment installed in the above-mentioned simultaneous DSC/WAXD/ SAXS measurement system, and heated up at $10^{\circ} \mathrm{C} /$ min, during which the 2-D WAXD and SAXS patterns were measured every $10 \mathrm{~s}$. As mentioned already, the WAXD patterns were measured using a FP detector, the efficiency of which was attained maximal for the $\mathrm{X}$-ray wave length of $0.1 \mathrm{~nm}$.

Figure 9 shows the 2-D WAXD and SAXS patterns measured for the uniaxially-oriented PVDF form I sample at $154.5^{\circ} \mathrm{C}$ and $164.6^{\circ} \mathrm{C}$. The WAXD pattern at $154^{\circ} \mathrm{C}$ corresponds to that of pure form I crystal. The SAXS was the four-points scattering pattern, indicating a tilt of stacked lamellae about $50^{\circ}$ from the draw axis. As the temperature as increased, the intensity of form I reflections became weaker. In the WAXD pattern taken at $164.7^{\circ} \mathrm{C}$, the new pattern was found to appear, which corresponded well to the X-ray diffraction pattern of PVDF form III, as pointed out by Tashiro et al. ${ }^{40}$ Here the contribution of amorphous halo was subtracted to show the pattern clearly. In Figure 10 the X-ray profiles along the $45^{\circ}$ and meridional directions, which were estimated from the 2-D WAXD patterns, are reproduced. The new reflections of the HT phase (form III) can be detected to appear before melt. The SAXS pattern was found to change also drastically from the four-points scattering pattern to the meridional two-points scattering pattern. Therefore the tilting angle of lamellae became almost zero, and the long period increased remarkably from $10 \mathrm{~nm}$ to $53 \mathrm{~nm}$. The half-width of the SAXS pattern

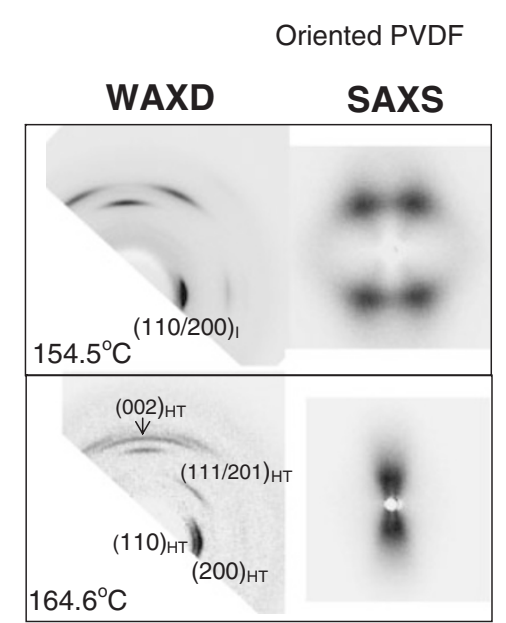

Figure 9. WAXD and SAXS patterns measured simultaneously for oriented PVDF sample at $154.5^{\circ} \mathrm{C}$ and $164.5^{\circ} \mathrm{C}$. The contribution of amorphous hallo was subtracted to show the WAXD patterns more clearly. The meridional direction is parallel to the oriented direction of sample. (a)

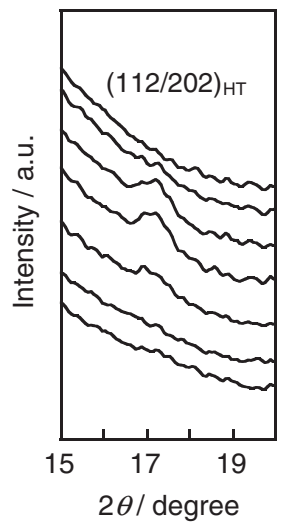

(b)

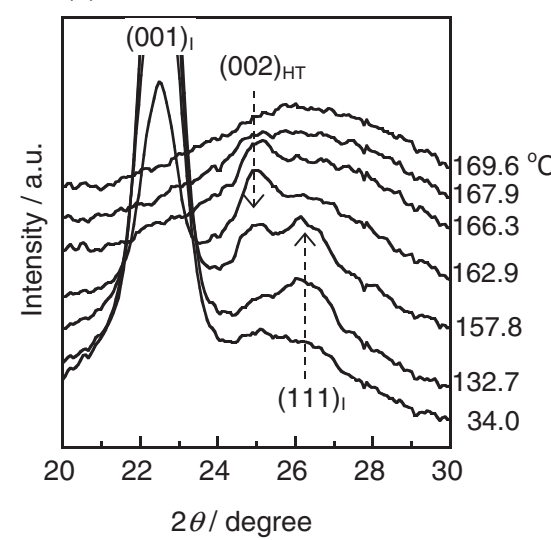

Figure 10. WAXD profiles estimated for the uniaxiallyoriented PVDF sample during heating process at $10^{\circ} \mathrm{C} / \mathrm{min}$. (a) A line profile at an azimuthal angel of 45 degree. (b) A line profile on the meridional direction.

became sharper along the equatorial direction or the direction perpendicular to the draw axis. This means that the lamellar size along the equatorial line increased drastically at the same timing as the remarkable increase of the long period along the draw direction. Figure 11 shows the temperature dependences of $\mathrm{X}$ ray reflection intensity estimated for form I and HT phase and that of the long period and lamellar tilting angle in comparison with the DSC thermogram. A shoulder detected in the lower temperature side of DSC peak corresponds to the phase transition from form I to HT phase.

In this way we succeeded to confirm the phase transition of PVDF form I occurring in the temperature region close to the melting point. This phenomenon 


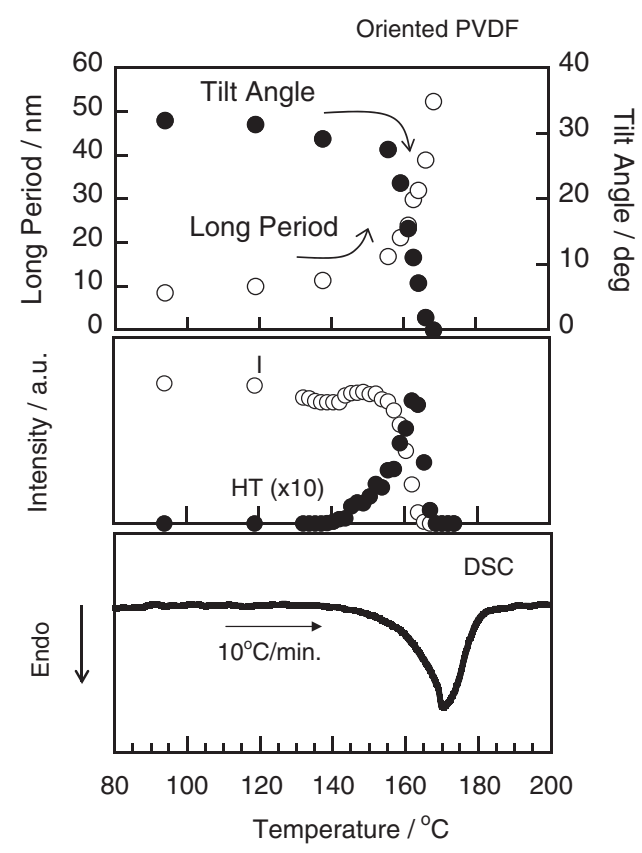

Figure 11. Temperature dependences of long period and tilting angle of stacked lamellae, peak intensity of the $(001)_{\mathrm{I}}$ and $(002)_{\mathrm{HT}}$ reflections and DSC thermogram measured for the uniaxially-oriented PVDF form I in the heating process at $10.0^{\circ} \mathrm{C} / \mathrm{min}$.

is very similar to that observed for uniaxially-oriented VDF $82 \%$ copolymer sample discussed in the previous section. It should be noticed here that the stacked lamellar structure changes remarkably from the tilted structure to the vertically standing form during this phase transition. This morphological change is essentially the same as that reported for VDF-TrFE copolymers of VDF 65 and $73 \% .{ }^{38}$ Such a drastic morphological change occurring at the same timing as the crystalline phase transition can be detected not only for a series of VDF copolymer but also for many other polymers such as nylon, syndiotactic polystyrene, poly(lactic acid) and so on. ${ }^{41-45}$ In all these cases the thermally-activated molecular motion of polymer chains along the chain axis causes the increase in lamellar thickness and the standing up of thicker lamellae along the draw direction, as illustrated in Figure 6. We may say that the drastic change in lamellar morphology linked with the crystalline phase transition occurring in a HT region close to the melting point is a universal phenomenon for many crystalline polymers.

\section{CONCLUSIONS}

In the present paper we have reported the development of equipment for the DSC/WAXD/SAXS simultaneous measurement for polymeric materials using a strong synchrotron X-ray radiation, which was built up successfully at the BL40B2 in SPring-8.
This 2-D WAXD/SAXS measurement system can be combined with the various equipments such as a Raman scattering, light scattering, etc. and makes it possible to trace the structural change viewed in a short time. We demonstrated the usefulness of this system by describing the experimental data about the phase transitions of VDF $82 \%$ copolymer and PVDF form I occurring in the HT region immediately below the melting point. These experiments were difficult for a long time as long as the conventional X-ray diffraction system of laboratory level was used. The newly developed DSC/WAXD/SAXS measurement system has leaded us to the success in detecting these phase transition phenomena satisfactorily. As a result, we have been able to establish the important universal phenomenon common to many crystalline polymers: the morphological change of stacked lamellae occurs in strong association with the crystalline phase transition. This system will be improved regarding data collection and analysis.

We hope that many users in the research field of polymer science and engineering can use actively this simultaneous measurement system developed newly at the BL40B2 in SPring- 8 for various kinds of research themes.

Acknowledgment. The authors wish to thank Daikin Co. and Kureha Co. for supplying VDF 82\% copolymer sample and high-molecular-weight PVDF sample, respectively. The synchrotron radiation experiments were performed at the BL40B2 in SPring-8 with the approval of the Japan Synchrotron Radiation Research Institute (JASRI) (Proposal No. 2006B1100. and 2007A1321.) H. M. wishes to thank Dr. Kazuki Ito, RIKEN for his kind discussion in the development of the simultaneous measurement system in SPring-8.

\section{REFERENCES}

1. K. Tashiro and S. Sasaki, Prog. Polym. Sci. 28, 451 (2003).

2. K. Tashiro, S. Kariyo, A. Nishimori, T. Fujii, S. Saragai, S. Nakamoto, T. Kawaguchi, A. Matsumoto, and O. J. Rangsiman, J. Polym. Sci., Part B: Polym. Phys. 40, 495 (2002).

3. B. Chu and B. S. Hasio, Chem. Rev., 101, 1727 (2001).

4. F. S. Bates, K. A. Koppi, M. Tirrell, K. Almdal, and K. Moritensen, Macromolecules, 27, 5934 (1994).

5. W. Bras, G. E. Derbyshire, D. Boss, J. Cooke, M. J. A. Elwell, B. E. Komanschek, S. Naylor, and A. J. Ryan, Science 267, 5200 (1995).

6. D. Cookson, N. Kirby, R. Knott, M. Leea, and D. Schultza, J. Synchrotron Rad., 13, 440, (2006).

7. V. Urban, P. Panine, C. Ponchut, P. Boesecke, and T. Narayanan, J. Appl. Crystallogr., 36, 809 (2003).

8. W. Bras, G. E. Derbyshire, A. Devine, S. M. Clark, J. 
Cooke, B. E. Komanschek, and A. J. Ryan, J. Appl. Crystallogr., 28, 26 (1995).

9. W. Bras, I. P. Dolbnya, D. Detollenaere, R. van Tol, M. Malfois, G. N. Greaves, A. J. Ryanc, and E. Heeley, J. Appl. Crystallogr., 36, 791 (2003).

10. R. J. Cernik, P. Barnes, G. Bushnell-Wye, A. J. Dent, G. P. Diakun, J. V. Flaherty, G. N. Greaves, E. L. Heeley, W. Helsby, S. D. M. Jacques, J. Kay, T. Rayment, A. Ryan, C. C. Tanga, and N. J. Terrilla, J. Synchrotron Rad., 11, 163 (2004).

11. Y. H. Lai, Y. S. Sun, U. S. Jeng, J. M. Lin, T. L. Lin, H. S. Sheu, W. T. Chuang, Y. S. Huang, C. H. Hsu, M. T. Lee, H. Y. Lee, K. S. Liang, A. Gabriel, and M. H. J. Koch, J. Appl. Crystallogr., 39, 871 (2006).

12. S. Rastogi, J. G. P. Goosens, and P. J. Lemstra, Macromolecules, 31, 2983 (1998).

13. S. Okamoto, K. Saijo, and T. Hashimoto, Macromolesules, 27, 3753 (1994).

14. S. Okamoto, K. Yamamoto, K. Komura, S. Hara, I. Akiba, K. Sakurai, A. Koyama, M. Nomura, and S. Sakurai, J. Macromol. Sci., Part B Phys. 43, 279 (2004).

15. Y. Nozue, Y. Shinohara, Y. Ogawa, T. Sakurai, H. Hori, T. Kasahara, N. Yamaguchi, N. Yagi, and Y. Amemiya, Macromolecules, 40, 2036 (2007).

16. S. Abe and H. Takahashi, J. Appl. Crystallogr., 36, 515 (2003).

17. H. Takahashi, S. Ueno, and C. Katagiri, J. Appl. Crystallogr., 36, 545 (2003).

18. K. N. Krueger and H. G. Zachmann, Macromolecules, 26, 5202 (1993).

19. G. K. Bryant, H. F. Gleeson, A. J. Ryana, J. P. A. Fairclough, D. Bogg, J. G. P. Goossens, and W. Bras, Rev. Sci. Instrum., 69, 2114 (1998).

20. K. Ito, T. Fujisawa, and T. Iwata, AIP Conference Proceedings, 879, 1160 (2006).

21. N. Yagi and K. Inoue, J. Appl. Crystallogr., 40, s437 (2007).

22. K. Inoue, T. Oka, K. Miura, and N. Yagi, AIP Conference Proceedings, 705, 336 (2004).

23. Y. Amemiya, K. Ito, N. Yagi, Y. Asano, K. Wakabayashi, T. Ueki, and T. Endo, Rev. Sci. Instrum. 66, 2290 (1995).

24. I. Hatta, H. Takahashi, S. Matsuoka, and Y. Amemiya, Thermochim. Acta, 253, 149 (1995).
25. H. Yoshida, K. Watanabe, R. Watanabe, and T. Iyoda, Trans. Mater. Res. Soc., Jpn., 29, 861 (2004).

26. M. Higami, S. Ueno, T. Segawa, K. Iwanami, and K. Sato, J. Am. Oil Chem. Soc., 80, 731 (2003).

27. K. Tashiro, in "Ferroelectric polymers," H. S. Nalwa Ed., New York, Marcel Dekker, Chapter 2, 1995.

28. A. J. Lovinger, Jpn. J. Appl. Phys. Suppl, 24, 18 (1985).

29. K. Tashiro, R. Tanaka, K. Ushitora, and M. Kobayashi, Ferroelectrics, 171, 145 (1995).

30. J. B. Lando, H. G. Olf, and A. Peterlin, J. Polym. Sci., Part A: Polym. Chem., 4, 941 (1966).

31. R. Hasegawa, Y. Takahashi, Y. Chatani, and H. Tadokoro, Polym. J., 3, 600 (1971).

32. K. Tashiro, K. Takano, M. Kobayashi, Y. Chatani, and H. Tadokoro, Polymer, 25, 195 (1984).

33. B. L. Farmer, A. J. Hopfinger, and J. B. Lando, J. Appl. Phys., 43, 4293 (1972).

34. N. Karasawa and W. A. Goddard III, Macromolecules, 25, 7268 (1992).

35. N. Hasegawa, M. Kobayashi, and K. Tashiro, Polym. J., 3, 591 (1971).

36. K. Tashiro, Y. Abe, and M. Kobayashi, Ferroelectrics, 171, 281 (1995).

37. K. Tashiro and M. Kobayashi, Polym. Prepr. Jpn., 34, 2241 (1985).

38. K. Tashiro and R. Tanaka, Polymer, 47, 5433 (2006).

39. K. Tashiro, K. Takano, M. Kobayashi, Y. Chatani, and H. Tadokoro, Polymer, 24, 199 (1983).

40. K. Tashiro, K. Takano, M. Kobayashi, Y. Chatani, and H. Tadokoro, Polym. Bull., 10, 464 (1983).

41. K. Tashiro, Chin. J. Polym. Sci., 25, 73 (2007).

42. K. Tashiro, K. Takeuchi, Y. Ohta, M. Hanesaka, T. Hashida, Y. Yoshioka, and C. Ramesh, Macromol. Symp., 242, 250 (2006).

43. E. B. Gowd, N. Shibayama, and K. Tashiro, Macromol. Symp., 242, 257 (2006).

44. K. Tashiro, E. B. Gowd, and M. Hanesaka, presented at the SAS2006 Proceedings, Kyoto International Conference Hall, Kyoto, Japan, July 9-13, 2006.

45. J. Zhang, K. Tashiro, H. Tsuji, and A. J. Domb, Polym. Prepr., Jpn., 56, 686 (2007). 